\title{
Threshold Dynamics of a Diffusive Herpes Model Incorporating Fixed Relapse Period in a Spatial Heterogeneous Environment
}

\author{
Yueming Lu, ${ }^{1}$ Wei Yang, ${ }^{2}$ and Desheng Ji $\mathbb{D}^{2}$ \\ ${ }^{1}$ College of Science, Harbin University of Science and Technology, Harbin 150080, China \\ ${ }^{2}$ School of Mathematical Science, Heilongjiang University, Harbin 150080, China \\ Correspondence should be addressed to Desheng Ji; 2014025@hlju.edu.cn
}

Received 22 May 2021; Revised 11 December 2021; Accepted 13 December 2021; Published 30 December 2021

Academic Editor: M. De Aguiar

Copyright $\odot 2021$ Yueming Lu et al. This is an open access article distributed under the Creative Commons Attribution License, which permits unrestricted use, distribution, and reproduction in any medium, provided the original work is properly cited.

\begin{abstract}
In this paper, we aim to establish the threshold-type dynamics of a diffusive herpes model that assumes a fixed relapse period and nonlinear recovery rate. It turns out that when considering diseases with a fixed relapse period, the diffusion of recovered individuals will lead to nonlocal recovery term. We characterize the basic reproduction number, $\mathfrak{R}_{0}$, for the model through the next generation operator approach. Moreover, in a homogeneous case, we calculate the $\mathfrak{R}_{0}$ explicitly. By utilizing the principal eigenvalue of the associated eigenvalue problem or equivalently by $\mathfrak{R}_{0}$, we establish the threshold-type dynamics of the model in the sense that the herpes is either extinct or close to the epidemic value. Numerical simulations are performed to verify the theoretical results and the effects of the spatial heterogeneity on disease transmission.
\end{abstract}

\section{Introduction}

During the past three decades, the incidence of herpes has increased in the developing countries [1]. One typical feature of herpes is that the virus will be reactivated and reactivated periodically by close physical or sexual contact, leading to a relapse period of infectiousness (see, e.g., [1-5]). Mathematical models have been used to explore the transmission of herpes. It is highlighted in [5] that an ordinary differential equations (ODEs) compartment model for herpes is also suitable for pseudorabies in pigs [6]. In [5], the population was divided into three disjoint classes, that is, susceptible individuals (who have not previously been exposed to the virus), infective individuals (who have been infected and shed the virus), and recovered (or latency) individuals (who have previously been infected with the virus but have not shed the virus), denoted by $S(t), I(t)$, and $R(t)$, respectively. Considering that the relapse phenomenon occurs when the virus is reactivated, they used a relapse term from recovered class to infective class to describe a disease with relapse, giving the Susceptible-Infective-Recovered-Infective (SIRI) model. With the standard incidence rate, $\boldsymbol{R}_{0}$ is determined as the sharp threshold for determining whether the herpes is extinct or close to the epidemic value. The ODEs model in [5] was further extended to the more with general incidence function in [4], and similar threshold results were obtained. Subsequently, Blower et al. [3] formulated a model to investigate how much resistance of herpes will be produced when the rate of antiviral treatment is enlarged. For more different herpes transmission models, we refer to [2].

Unlike the ODEs models for herpes that the relapse period was assumed to obey negative exponential distribution, van den Driessche and Zou et al. [7] utilized a more general relapse distribution $P(t)$ to explore the results of distinct settings on the relapse period, where $P(t)$ stands for the proportion of recovered individuals still remaining in recovered class after recovery. In particular, the authors took a step function distribution for the relapse period and obtained a delay differential equations (DDEs) model for herpes. They also found that there is no sustained oscillatory solutions. After determining $\mathfrak{R}_{0}$, the threshold-type results of the model were also addressed.

The aforementioned models are for a spatially homogeneous environment, meaning that only ODE and DDEs 
models are involved. In recent years, spatial-temporal dynamics of infectious diseases governed by the reactiondiffusion models have attracted many researchers. The spatial heterogeneity $(\mathrm{SH})$ and diffusion play important roles in disease transmission. Under different infection mechanisms, some new insights in disease control and new phenomenon in disease spread will be obtained; see, for instance, [8-13]. It is found in [8] that $\mathrm{SH}$ would increase the risk of influenza transmission so that the $\mathrm{SH}$ of the recovery rate and transmission rate must be increased for controlling the influenza transmission. In [9], the authors proposed a spatial nonlocal diffusive model with delay and no-flux boundary condition. Here the nonlocal delay is caused by introducing a fixed incubation period in a continuous bounded domain. By utilizing the classical theory, the threshold-type dynamics are determined by $\Re_{0}$. Here, $\Re_{0}$ was achieved by the spectral radius of the next generation operator. In a homogeneous case that all model parameters are constant, $\boldsymbol{R}_{0}$ can be explicitly obtained. Besides, another method of calculating $\mathfrak{R}_{0}$ in one-dimensional space was also presented in [9]. In a recent work [14], the authors studied the dual-functionality of physical contacts driven via variations of individual spatial behavior and provided insights on mechanisms that generate spatial heterogeneity. By using an epidemic model with nonlocal delay and logistic growth, the authors in [15] studied the dynamics of model and investigated how nonlocal delay and logistic growth affect the disease transmission. It was advocated in [16] that the transitions between patterns are an emergent property in spatial epidemics that can serve as a potential trend indicator of disease spread when considering the spread of diseases in both time and space. In [17], the authors studied the existence and nonexistence of the traveling wave solutions for the model with spatial structure.

The authors in [18] studied the local and global longterm dynamics of the Banana Black Sigatoka Disease with delay and seasonality. In [19], the spatial-temporal characteristics and effective control measures of brucellosis transmission are investigated. Very recently, a humanvector malaria transmission model incorporating age, time since infection, and waning immunity was studied in [20], where the well-posedness of the model, the existence of endemic equilibria, and the effect of the above structural variables on key important epidemiological traits of the human-vector association are demonstrated. $\mathrm{Wu}$ and $\mathrm{Zhao}$ [21] studied a nonlocal and delayed diffusive HIV latent infection model with spatial heterogeneity and the effects of spatial heterogeneity and delays on viral dynamics are investigated. With a simple mathematical model, Gaythorpe and Adams [22] examine how demographic and environmental heterogeneities, population behavior, and behavioural change respond to the provision of facilities, and they also studied how to reduce epidemic size and endemic prevalence by the optimal configurations of limited numbers of facilities.

This paper is also inspired by nonlocal and delayed reaction-diffusion systems in bounded domains $[9,10]$, which discussed the common influence of incubation period and $\mathrm{SH}$ on the spatial spread of disease. When considering infectious diseases with a fixed incubation period, the migration of infected individuals will lead to nonlocal infection $[9,10]$. We assume that the host population lives in a bounded spatial habitat $\Omega$ with smooth boundary $\partial \Omega$. At time $t$ and location $x$, we denote by $S(t, x), I(t, x)$, and $R(t, x)$ the densities of susceptible individuals, infectious individuals, and recovered individuals, respectively. In the absence of disease, susceptible individuals would approach a steady state; that is, $S(t, x)$ will be governed by the following equation:

$$
\begin{cases}\frac{\partial S}{\partial t}=d_{S} \triangle S+\lambda(x)-\mu_{S}(x) S, & t \geq 0, x \in \Omega, \\ \frac{\partial S(t, x)}{\partial n}=0, & t>0, x \in \partial \Omega,\end{cases}
$$

where $d_{S}>0$ represents the dispersal rate of susceptible individuals and $\Delta$ is the Laplacian operator. With respect to space variable $x, \lambda(x)$ and $\mu_{S}(x)$, respectively, represent the recruitment rate and death rate of susceptible individuals depending on spatial variable $x$. If infection occurs, we adopt disease transmission functions as Beddington-DeAngelis functional response and use the following equations to describe the interactions between susceptible and infectious individuals:

$$
\begin{cases}\frac{\partial S}{\partial t}=d_{S} \Delta S+\lambda(x)-\mu_{S}(x) S-\frac{\beta(x) S I}{1+a(x) S+b(x) I}, & t>0, x \in \Omega, \\ \frac{\partial I}{\partial t}=d_{I} \Delta I+\frac{\beta(x) S I}{1+a(x) S+b(x) I}-\left(\mu_{I}(x)+k(x)\right) I, & t>0, x \in \Omega, \\ \frac{\partial S(t, x)}{\partial n}=\frac{\partial I(t, x)}{\partial n}=0, & t>0, x \in \partial \Omega,\end{cases}
$$

where $d_{I}>0$ represents the diffusion rate of infective individuals. $n$ is the outward normal along $\partial \Omega$. $\beta(x)$ is the transmission rate between susceptible and infectious individuals. Note that the diffusion rate may be different from susceptible to infectious individuals depending on the disease. Here, the Beddington-DeAngelis functional response can be considered as the extensions of Holling's type II and saturation functional response, allowing a behavioural state, namely, 'mutual interference with competitors.' $a(x)$ measures the susceptible individuals interference. $b(x)$ determine how fast the transmission/infection rate will approach saturation [23]. $\mu_{I}(x)$ and $k(x)$, respectively, represent the death rate and removed rate of infective individuals. All these functions are positive and Hölder continuous functions, which allow the SH due to the fact that spatial habitat environment is always different.

We introduce age $a$ representing the relapse age to recovered individuals. With relapse age $a$, time $t$, and location $x$, we denote by $R(t, a, x)$ the density of recovered individuals. By the standard arguments as in [24], we suppose recovered individuals are dominated by 


$$
\begin{cases}\frac{\partial R(t, a, x)}{\partial t}+\frac{\partial R(t, a, x)}{\partial a}=d_{R} \Delta R(t, a, x)-\left(\mu_{R}(x)+r(a, x)\right) R(t, a, x), & t>0, x \in \Omega, \\ R(t, 0, x)=\frac{k(x) I(t, x)}{1+m(x) I(t, x)}, & t>0, x \in \Omega, \\ \frac{\partial R(t, a, x)}{\partial n}=0, & t>0, x \in \partial \Omega,\end{cases}
$$

where $d_{R}>0$ stands for the dispersal rate of recovered individuals. $\mu_{R}(x)$ is the death rate. $r(a, x)$ represent the relapse rate from recovered individuals to infectious individuals with relapse age $a$ and location $x . m(x)$ measures how fast the recovery rate of infectious individuals will approach saturation.

Biologically, we suppose that $\tau>0$ is the average (fixed) relapse period, denoted by

$R_{e}(t, x)=\int_{0}^{\tau} R(t, a, x) \mathrm{d} a, R_{r}(t, x)=\int_{\tau}^{\infty} R(t, a, x) \mathrm{d} a$.

Assume that the function $r(a, x)$ satisfies

$$
r(a, x)= \begin{cases}0, & \text { for } a \in[0, \tau] \text { and } x \in \Omega, \\ r(x), & \text { for } a \in[\tau, \infty) \text { and } x \in \Omega .\end{cases}
$$

We calculate the derivative of $R_{e}(t, x)$ and $R_{r}(t, x)$ by using (3) and (5) to get

$$
\frac{\partial R_{e}}{\partial t}=d_{R} \triangle R_{e}-\mu_{R}(x) R_{e}+R(t, 0, x)-R(t, \tau, x),
$$

and

$$
\frac{\partial R_{r}}{\partial t}=d_{R} \triangle R_{r}-\left(\mu_{R}(x)+r(x)\right) R_{r}+R(t, \tau, x)-R(t, \infty, x),
$$

respectively. Assuming that $R(t, \infty, x)=0$, this means that $\partial R_{e}(t, x) / \partial t$ and $\partial R_{r}(t, x) / \partial t$ will be obtained if $R(t, \tau, x)$ is known. To this end, we integrate (3) along the characteristic line $t=a+r$ by introducing $\rho(r, a, x)=R(a+r, a, x), r \geq 0$. Hence, for $a \in[0, \tau]$, we directly have

$$
\frac{\partial \rho}{\partial a}=\left[\frac{\partial R}{\partial t}+\frac{\partial R}{\partial a}\right]_{t=a+r}=d_{R} \Delta \rho-\mu_{R}(x) \rho,
$$

with

$$
\rho(r, 0, x)=R(r, 0, x)=\frac{k(x) I}{1+m(x) I}
$$

It then follows that

$$
\rho(r, a, x)=\int_{\Omega} G(a, x, y) \frac{k(y) I(r, y)}{1+m(y) I(r, y)} \mathrm{d} y,
$$

where $G$ stands for the Green function to the operator $d_{R} \Delta-\left(\mu_{R}(\cdot)+r(x)\right)$. Changing the variable $a$ by $\tau$ (hence $r=t-\tau)$, we yield

$$
R(t, \tau, x)=\rho(t-\tau, \tau, x)=\int_{\Omega} G(\tau, x, y) \frac{k(y) I(t-\tau, y)}{1+m(y) I(t-\tau, y)} \mathrm{d} y, \quad \forall t \geq \tau
$$

Putting (11) into (6) and (7), respectively, and noting that $R_{e}(t, x)$ is decoupled from $S, I$, and $R_{r}$ equation, we arrive at the following system: 


$$
\begin{cases}\frac{\partial S}{\partial t}=d_{S} \Delta S+\lambda(x)-\mu_{S}(x) S-\frac{\beta(x) S I}{1+a(x) S+b(x) I}, & t>0, x \in \Omega, \\ \frac{\partial I}{\partial t}=d_{I} \Delta I+\frac{\beta(x) S I}{1+a(x) S+b(x) I}-\left(\mu_{I}(x)+k(x)\right) I+r(x) R_{r}, & t>0, x \in \Omega, \\ \frac{\partial R_{r}}{\partial t}=d_{R} \Delta R_{r}-\left(\mu_{R}(x)+r(x)\right) R_{r}+\int_{\Omega} G(\tau, x, y) \frac{k(y) I(t-\tau, y)}{1+m(y) I(t-\tau, y)} \mathrm{d} y, & t>0, x \in \Omega, \\ \frac{\partial S(t, x)}{\partial n}=\frac{\partial I(t, x)}{\partial n}=\frac{\partial R_{r}(t, x)}{\partial n}=0, & t>0, x \in \partial \Omega .\end{cases}
$$

For convenience, we denote

$$
\left(u_{1}, u_{2}, u_{3}\right)=\left(S, I, R_{r}\right),\left(d_{1}, d_{2}, d_{3}\right)=\left(d_{S}, d_{I}, d_{R}\right) \text {. }
$$

Then in the sequel, we will study the following system:

$$
\begin{cases}\frac{\partial u_{1}}{\partial t}=d_{1} \Delta u_{1}+\lambda(x)-\mu_{S}(x) u_{1}-\frac{\beta(x) u_{1} u_{2}}{1+a(x) u_{1}+b(x) u_{2}}, & t>0, x \in \Omega \\ \frac{\partial u_{2}}{\partial t}=d_{2} \Delta u_{2}+\frac{\beta(x) u_{1} u_{2}}{1+a(x) u_{1}+b(x) u_{2}}-\left(\mu_{I}(x)+k(x)\right) u_{2}+r(x) u_{3}, & t>0, x \in \Omega \\ \frac{\partial u_{3}}{\partial t}=d_{3} \Delta u_{3}-\left(\mu_{R}(x)+r(x)\right) u_{3}+\int_{\Omega} G(\tau, x, y) \frac{k(y) u_{2}(t-\tau, y)}{1+m(y) u_{2}(t-\tau, y)} d y, & t>0, x \in \Omega \\ \frac{\partial u_{1}(t, x)}{\partial n}=\frac{\partial u_{2}(t, x)}{\partial n}=\frac{\partial u_{3}(t, x)}{\partial n}=0, & t>0, x \in \partial \Omega\end{cases}
$$

We arrange the rest of this paper as follows. Section 2 is devoted to the well-posedness of system (14). We follow the standard procedures in [25] to define $\boldsymbol{R}_{0}$ for (14) by the next generation operator approach in Section 3. Moreover, in a homogeneous case, we calculate $\mathfrak{R}_{0}$ explicitly. In Section 4 , $\mathfrak{R}_{0}$ will be verified that it takes a role of a threshold index for herpes extinction and persistence.

\section{Well-Posedness of System}

For convenience, we introduce the spaces and notations used in this paper.

(i) Denote $\mathbb{X}:=C\left(\bar{\Omega}, \mathbb{R}^{3}\right)$ and the positive cone of $\mathbb{X}$ is denoted by $\mathbb{X}^{+}:=C\left(\bar{\Omega}, \mathbb{R}_{+}^{3}\right)$

(ii) Denote $C=C([-\tau, 0], \mathbb{X})$ and its positive cone is denoted by $C^{+}:=C\left([-\tau, 0], \mathbb{X}^{+}\right)$, associated with the norm $\|\phi\|=\max _{\theta \in[-\tau, 0]}\|\phi(\theta)\|_{\mathbb{X}}$, for $\tau \geq 0$

(iii) Denote by $u_{t} \in \stackrel{\theta \in[-\tau, 0]}{C}$ the $u_{t}(\theta)=u(t+\theta), \theta \in[-\tau, 0]$, where $u:[-\tau, \sigma) \longrightarrow \mathbb{X}$ for $\sigma>0$

(iv) Denote $\mathbb{Y}:=C(\bar{\Omega}, \mathbb{R})$ and its positive cone is denoted by $\bigvee^{+}:=C\left(\bar{\Omega}, \mathbb{R}_{+}\right)$ (v) Denote by $T_{i}(t)(i=1,2,3): \mathbb{Y} \longrightarrow \mathbb{Y}, t \geq 0$, the strongly continuous semigroups with respect to the operators $d_{1} \Delta-\mu_{S}(\cdot), d_{2} \Delta-\left(\mu_{I}(\cdot)+k(\cdot)\right)$, and $d_{3} \Delta-\left(\mu_{R}(\cdot)+r(\cdot)\right)$, associated with no-flux boundary condition, respectively

(vi) Denote $\mathbb{E}:=C([-\tau, 0], \mathbb{Y}) \times \mathbb{Y}$ and its positive cone is denoted by $\mathbb{E}^{+}:=C\left([-\tau, 0], \mathbb{Y}^{+}\right) \times \mathbb{Y}^{+}$

Let $u(t, \cdot)=\left(u_{1}(t, \cdot), u_{2}(t, \cdot), u_{3}(t, \cdot)\right)$ be the solution corresponding to initial data $u_{0}=\left(u_{1}^{0}(\cdot), u_{2}^{0}(\cdot), u_{3}^{0}(\cdot)\right)$. Let $F=\left(F_{1}, F_{2}, F_{3}\right): C^{+} \longrightarrow \mathbb{Y}$ be defined as

$$
\left\{\begin{array}{l}
F_{1}(\phi)(\cdot)=\lambda(x)-\frac{\beta(\cdot) \phi_{1}(0, \cdot) \phi_{2}(0, \cdot)}{1+a(\cdot) \phi_{1}(0, \cdot)+b(x) \phi_{2}(0, \cdot)} \\
F_{2}(\phi)(\cdot)=\frac{\beta(\cdot) \phi_{1}(0, \cdot) \phi_{2}(0, \cdot)}{1+a(\cdot) \phi_{1}(0, \cdot)+b(\cdot) \phi_{2}(0, \cdot)}+r(\cdot) \phi_{3}(0, \cdot) \\
F_{3}(\phi)(\cdot)=\int_{\Omega} G(\tau, \cdot, y) \frac{k(y) \phi_{2}(-\tau, y)}{1+m(y) \phi_{2}(-\tau, y)} \mathrm{d} y
\end{array}\right.
$$


$\forall x \in \bar{\Omega}, \phi=\left(\phi_{1}, \phi_{2}, \phi_{3}\right)^{T} \in C^{+}$. It allows us to rewrite (14) as

$$
\left\{\begin{array}{l}
u=\tilde{u}(t) \phi+\int_{0}^{t} \tilde{u}(t-s) F\left(u_{t}(s)\right) \mathrm{d} s, t>0 \\
u_{0}=\phi \in C^{+},
\end{array},\right.
$$

where $\tilde{u}(t)=\left(T_{1}(t), T_{2}(t), T_{3}(t)\right)^{T}$. According to [26] [Section 7.1 and Corollary 7.2.3]), we obtain that $T_{1}(t), T_{2}(t)$, and $T_{3}(t)$ are compact and strongly positive on $\mathbb{Y}$. Further, for small enough $\sigma>0$, we have

$$
\begin{aligned}
\phi(0, \cdot)+\sigma F(\phi)(\cdot) & =\left(\begin{array}{c}
\phi_{1}(0, \cdot)+\sigma\left(\lambda(\cdot)-\frac{\beta(\cdot) \phi_{1}(0, \cdot) \phi_{2}(0, \cdot)}{1+a(\cdot) \phi_{1}(0, \cdot)+b(\cdot) \phi_{2}(0, \cdot)}\right) \\
\phi_{2}(0, \cdot)+\sigma\left(\frac{\beta(\cdot) \phi_{1}(0, \cdot) \phi_{2}(0, \cdot)}{1+a(\cdot) \phi_{1}(0, \cdot)+b(\cdot) \phi_{2}(0, \cdot)}+r(\cdot) \phi_{3}(0, \cdot)\right) \\
\phi_{3}(0, \cdot)+\sigma\left(\int_{\Omega} G(\tau, \cdot, y) \frac{k(y) \phi_{2}(-\tau, y)}{1+m(y) \phi_{2}(-\tau, y)} \mathrm{d} y\right),
\end{array}\right) \\
& \left(\phi _ { 1 } ( 0 , \cdot ) \left(1-\sigma \frac{\bar{\beta}}{\underline{b}) \phi_{2}(0, \cdot) \phi_{3}(0, \cdot)},\right.\right.
\end{aligned}
$$

where $\bar{\beta}=\max _{x \in \bar{\Omega}} \beta(x)$ and $\underline{b}=\min _{x \in \bar{\Omega}} b(x)$. It follows that

$$
\lim _{\sigma \longrightarrow 0^{+}} \frac{1}{\sigma} \operatorname{dist}(\phi(0)+\sigma F(\phi), \mathbb{X})=0, \forall \phi \in C^{+} .
$$

It then follows from [27] [Corollary 4] and [28] [Corollary 8.1.3]) that, for any $u_{0}=\phi \in C^{+}$, there exists a unique noncontinuable mild solution $u(t, \phi)$ on $\left[0, t_{\max }\right)$. Moreover, $u(t, \phi) \in \mathbb{X}, \forall t \in\left[0, t_{\max }\right)$ and $u(t, \phi)$ is a classical solution of (14) for $t>\tau$.

Before going into details, we first introduce a useful lemma; see also in [10] Lemma 1.

Lemma 1. Consider the following system:

$$
\begin{cases}\frac{\partial \omega}{\partial t}=d_{1} \Delta \omega+\lambda(x)-\mu_{S}(x) \omega, & t>0, x \in \Omega, \\ \frac{\partial \omega(t, x)}{\partial n}=0, & t>0, x \in \partial \Omega .\end{cases}
$$

Then system (19) admits a unique and global asymptotic stable positive steady state (PSS) $E(x)$ in $\mathbb{Y}^{+}$. Moreover, if both $\lambda$ and $\mu_{S}$ are positive constants, then $U=v / \mu_{S}$.

Notice that $\partial u_{1} / \partial t \leq d_{1} \Delta u_{1}+\lambda(x)-\mu_{S}(x) u_{1}$. By the comparison theorem,

$\lim \sup u_{1}(t, x) \leq \lim \sup \omega(t, x)=E(x)$, uniformly for $x \in \bar{\Omega}$.
Hence, there exists $K>0$ such that

$$
\left\|u_{1}(t, x)\right\| \leq K, \quad t \geq 0,
$$

where $K$ depends on initial data.

Theorem 1. For each $\phi \in C^{+}$, (14) admits a unique solution $u(t, \phi)$ on $[0, \infty)$. Let $\Phi(t)=u_{t}(\cdot): C^{+} \longrightarrow C^{+}, t \geq 0$ be the solution semiflow of (14), defined by $(\Phi(t) \phi)$ $(\theta, x)=u(t+\theta, x, \phi), \forall \theta \in[-\tau, 0], x \in \bar{\Omega}$, which has a global compact attractor.

Proof. It follows from (20) that $u_{1}(t, \phi)$ is bounded on $\left[0, t_{\max }\right)$. Then there exists $Q>0$ such that $u_{3}$-equation of (14) is dominated by

$$
\begin{cases}\frac{\partial \omega}{\partial t}=d_{3} \Delta \omega-\left(\mu_{R}(x)+r(x)\right) \omega+Q, & t>0, x \in \Omega, \\ \frac{\partial \omega(t, x)}{\partial n}=0, & t>0, x \in \partial \Omega .\end{cases}
$$

By the comparison principle and Lemma $1, u_{3}(t, \phi)$ is bounded on $\left[0, t_{\max }\right)$. Similarly, $u_{2}(t, \phi)$ is bounded on $\left[0, t_{\max }\right)$. Hence, $u(t, \phi)=\left(u_{1}(t, \phi), u_{2}(t, \phi), u_{3}(t, \phi)\right)^{T}$ is bounded on $\left[0, t_{\max }\right)$, and hence $t_{\max }=+\infty$ for each $\phi \in C^{+}$. Therefore, the solution semiflow of system (14) is well defined.

For any $\phi \in C^{+}$, there exists $t_{1}>0$, and we have

$$
\left\{\frac{\partial u_{3}}{\partial t} \leq d_{3} \Delta u_{3}-\left(\mu_{R}(x)+r(x)\right) u_{3}+\frac{\bar{k}}{\underline{m}, t>t_{1}, x \in \Omega, \frac{\partial u_{3}(t, x)}{\partial n}=0, t>t_{1}, x \in \partial \Omega},\right.
$$


where $\bar{k}=\max _{x \in \bar{\Omega}} k(x), \underline{m}=\min _{x \in \bar{\Omega}} m(x)$. Again, from Lemma 1, there is a time $t_{2}(\phi)>t_{1}$ such that $u_{3}(t, \phi) \leq 2 \bar{k} / \underline{m}\left(\underline{\mu_{R}}+\underline{r}\right)$, $\forall t>t_{2}$, where $\underline{\mu_{R}}=\min _{x \in \bar{\Omega}}\left\{\mu_{R}(x)\right\}$ and $\underline{r}=\min _{x \in \bar{\Omega}}\{r(x)\}$. Further,

$$
\begin{cases}\frac{\partial u_{2}(t, x)}{\partial t} \leq d_{2} \Delta u_{2}(t, x)+\frac{\overline{\beta \lambda}}{\underline{b} \underline{\mu_{s}}}-\left(\mu_{I}(x)+k(x)\right) u_{2}(t, x)+2 \frac{\bar{r} \bar{k}}{\underline{m}\left(\underline{\mu_{R}}+\underline{r}\right)}, & t>t_{2}, x \in \Omega \\ \frac{\partial I(t, x)}{\partial n}=0, & t>t_{2}, x \in \partial \Omega\end{cases}
$$

where $\bar{\lambda}=\max \lambda(x)$ and $\mu_{s}=\min \mu_{s}(x)$. By Lemma 1 , there is $t_{2}(\phi)>^{x \in \bar{\Omega}} \quad$ such that $\quad x \in \bar{\Omega}(t, \phi) \leq 2 \overline{\beta \lambda} \underline{m} \quad\left(\underline{\left.\mu_{R}+\underline{r}\right)+}\right.$ $2 \underline{b} \underline{\mu_{S}} \bar{r} \bar{k} / \underline{b} \underline{\mu_{S}} \underline{m}\left(\underline{\mu_{R}}+\underline{r}\right)\left(\underline{\mu_{I}}+\underline{k}\right), \forall t>t_{2}$, where $\underline{\mu_{I}}=\underline{\min } \mu_{I}$ $(x)$ and $\underline{k}=\min _{x \in \bar{\Omega}} k(x)$. Hence, $\Phi(t): C^{+} \longrightarrow C^{+}$is point dissipative. Further from [28] [Theorem 2.1.8], $\Phi(t): C^{+} \longrightarrow C^{+}$is compact for any $t>\tau$. Hence, the last assertion directly follows from [29] [Theorem 3.4.8].

Lemma 2. For each $u_{0}=\phi \in C^{+}$, denote by $u(t, x, \phi)$ the solution of (14).

(i) If $u_{2}\left(t_{0}, \cdot, \phi\right) \neq 0$ for some $t_{0} \geq 0$, then $u_{2}\left(t_{0}, \cdot, \phi\right) \neq 0$ and $u_{2}(t, x, \phi)>0, \forall t>t_{0}, x \in \bar{\Omega}$.

(ii) $u_{1}(t, x, \phi)>0, \forall t>0, x \in \bar{\Omega}$ and

$$
\liminf _{t \rightarrow \infty} u_{1}(t, x) \geq \frac{\underline{\lambda}}{\bar{\mu}_{S}+\bar{\beta} / \underline{b}}, \text { uniformly for } x \in \bar{\Omega},
$$

where $\underline{\lambda}=\min _{x \in \bar{\Omega}} \lambda(x), \bar{\mu}_{S}=\max _{x \in \bar{\Omega}} \mu_{S}(x)$.

Proof. It is easy to see that $u_{2}$-equation satisfies

$$
\begin{cases}\frac{\partial u_{2}}{\partial t} \geq d_{2} \Delta u_{2}-\left(\bar{\mu}_{I}+\bar{k}\right) u_{2}, & t>0, x \in \Omega \\ \frac{\partial u_{2}(t, x)}{\partial n}=0, & t>0, x \in \partial \Omega,\end{cases}
$$

where $\bar{\mu}_{I}=\max _{x \in \bar{\Omega}} \mu_{I}(x)$. Then (i) holds directly from the comparison principle.

Denote by $\widehat{u}_{1}(t, x, \phi)$ the solution of

$\left\{\frac{\partial \widehat{u}_{1}}{\partial t} \geq d_{1} \Delta \widehat{u}_{1}+\lambda(x)-\left(\mu_{S}(x)+\frac{\bar{\beta}}{\underline{b}}\right) \widehat{u}_{1}, \frac{\partial \widehat{u}_{1}(t, x)}{\partial n}=0, \quad x \in \partial \Omega\right.$.

Then from Lemma 1 , and the comparison principle, $u_{1}(t, x, \phi) \geq \widehat{u}_{1}(t, x, \phi)>0, \forall t>0, x \in \bar{\Omega}$, and last assertion of (ii) directly follows. This completes the proof.

\section{Basic Reproduction Number}

By setting $u_{2}=0$, it is easy to find that the density of the susceptible individuals satisfies (19). Equation (14) possesses a disease-free steady state (DFSS), denoted by $(E(x), 0,0)$. Linearizing system (14) at DFSS,

$$
\begin{cases}\frac{\partial u_{2}}{\partial t}=d_{2} \Delta u_{2}+\left(\frac{\beta(x) E(x)}{1+a(x) E(x)}-\mu_{I}(x)-k(x)\right) u_{2}+r(x) u_{3}, & t>0, x \in \Omega \\ \frac{\partial u_{3}}{\partial t}=d_{3} \Delta u_{3}-\left(\mu_{R}(x)+r(x)\right) u_{3}+\int_{\Omega} G(\tau, x, y) k(y) u_{2}(t-\tau, y) \mathrm{d} y, & t>0, x \in \Omega \\ \frac{\partial u_{2}}{\partial n}=\frac{\partial u_{3}}{\partial n}=0, & t>0, x \in \partial \Omega\end{cases}
$$

which is a time-delayed and nonlocal linear system. In this circumstance, we first consider the following system: 


$$
\begin{cases}\frac{\partial u_{2}}{\partial t}=d_{2} \Delta u_{2}+\left(\frac{\beta(x) E(x)}{1+a(x) E(x)}-\mu_{I}(x)-k(x)\right) u_{2}+r(x) u_{3}, & t>0, x \in \Omega, \\ \frac{\partial u_{3}}{\partial t}=d_{3} \Delta u_{3}-\left(\mu_{R}(x)+r(x)\right) u_{3}+\int_{\Omega} G(\tau, x, y) k(y) u_{2}(t, y) d y, & t>0, x \in \Omega, \\ \frac{\partial u_{2}}{\partial n}=\frac{\partial u_{3}}{\partial n}=0, & t>0, \forall x \in \partial \Omega .\end{cases}
$$

The following nonlocal eigenvalue problem is obtained by inserting $u_{2}=e^{\lambda t} \psi(x)$ and $u_{3}=e^{\lambda t} \psi(x)$ into (29):

$$
\begin{cases}\lambda \psi_{1}(x)=d_{2} \Delta \psi_{1}(x)+\left(\frac{\beta(x) E(x)}{1+a(x) E(x)}-\mu_{I}(x)-k(x)\right) \psi_{1}(x)+r(x) \psi_{2}(x), & x \in \Omega, \\ \lambda \psi_{2}(x)=d_{3} \Delta \psi_{2}(x)-\left(\mu_{R}(x)+r(x)\right) \psi_{2}(x)+\int_{\Omega} G(\tau, x, y) k(y) \psi_{1}(y) \mathrm{d} y, & x \in \Omega, \\ \frac{\partial \psi_{1}(x)}{\partial n}=\frac{\partial \psi_{2}(x)}{\partial n}=0, & x \in \partial \Omega .\end{cases}
$$

A direct application of the result in [26] [Theorem 7.6.1] gives that (30) admits a principal eigenvalue $\lambda(E)$ equipped with a positive eigenvector.

We now focus our attention on system (28). For any $\psi \in \mathbb{E}^{+} /\{0\}$, denote by $u(t, \psi), t \geq 0$, the solution of $(28)$. The following claim is valid.

(i) $u_{2}(t, \psi)(x)>0$ and $u_{3}(t, \psi)(x)>0$ for all $x \in \bar{\Omega}$ and $t>\tau$. have

In fact, if $\psi_{1}(0, \cdot) \equiv 0$ or $\psi_{2}(0, \cdot) \equiv 0$, we then directly $u_{2}(t, \psi)(x)>0$, and $u_{3}(t, \psi)(x)>0$, for all $x \in \bar{\Omega}, t>\tau$, from parabolic maximum principle. If there is some $\theta_{0} \in(0, \tau)$ that $\psi_{1}\left(-\theta_{0}, \cdot\right) \equiv 0$, then $u_{3}\left(\tau-\theta_{0}, \psi\right) \equiv 0$. If $u_{3}\left(\tau-\theta_{0}, \psi\right) \equiv 0$, we have

$$
\frac{\partial u_{3}\left(\tau-\theta_{0}, x\right)}{\partial t}=\int_{\Omega} G(\tau, x, y) \frac{k(y) u_{2}\left(-\theta_{0}, y\right)}{1+m(y) u_{2}\left(-\theta_{0}, y\right)} \mathrm{d} y>0, \forall x \in \Omega .
$$

By $u_{3}(t, \psi) \geq 0, t \geq 0$, and $u_{3}\left(\tau-\theta_{0}, \psi\right)(x)=0, \forall x \in \bar{\Omega}$, which implies that $\partial u_{3}\left(\tau-\theta_{0}, x\right) / \partial t \leq 0$, a contradiction. This results in $u_{3}(t, \psi)(x)>0, \forall t>\tau-\theta_{0}, x \in \bar{\Omega}$. Similarly, $u_{2}(t, \psi)(x)>0, \forall t>\tau-\theta_{0}, x \in \bar{\Omega}$.

With the help of [30] [Theorem 2.2], we give the result on the following eigenvalue problem:

$$
\begin{cases}\lambda \psi_{1}(x)=d_{2} \Delta \psi_{1}(x)+\left(\frac{\beta(x) E(x)}{1+a(x) E(x)}-\mu_{I}(x)-k(x)\right) \psi_{1}(x)+r(x) \psi_{2}(x), & x \in \Omega, \\ \lambda \psi_{2}(x)=d_{3} \Delta \psi_{2}(x)-\left(\mu_{R}(x)+r(x)\right) \psi_{2}(x)+e^{-\lambda \tau} \int_{\Omega} G(\tau, x, y) k(y) \psi_{1}(y) \mathrm{d} y, & x \in \Omega, \\ \frac{\partial \psi_{1}(x)}{\partial n}=\frac{\partial \psi_{2}(x)}{\partial n}=0, & x \in \partial \Omega .\end{cases}
$$


Lemma 3. Eigenvalue problem (33) has a principal eigenvalue $\bar{\lambda}(E, \tau)$ with a strongly positive eigenvector. Moreover, $\bar{\lambda}(E, \tau)$ has the same sign as $\lambda(E), \tau \geq 0$.

Inspired by the standard procedures in [25], we next define $\mathfrak{R}_{0}$ for (14) by the next generation operator approach.

(i) Suppose that both the infective and the recovered individuals are near DFSS.

(ii) Introduce $\left(\psi_{2}(x), \psi_{3}(x)\right)^{T}$ as the spatial initial distribution of the infective and recovered individuals.

(iii) At time $t$, denote by $\mathbb{S}(t) \psi:=\left(T_{2}(t) \psi_{2}, T_{3}(t) \psi_{3}\right)^{T}$ the remaining distribution of infective and recovered individuals.

(iv) $\forall \psi \in \mathbb{Y} \times \mathbb{Y}$, let

$$
\mathbb{V}(\psi)(x)=\left(V_{1}(\psi)(x), V_{2}(\psi)(x)\right), x \in \bar{\Omega},
$$

where

$$
V_{1}(\psi)(x)=\frac{\beta(x) E(x)}{1+a(x) E(x)} \psi_{2}(x)+r(x) \psi_{3}(x),
$$

and

$$
V_{2}(\psi)(x)=\int_{\Omega} G(\tau, x, y) k(y) \psi_{2}(y) \mathrm{d} y .
$$

As a result, $\mathbb{V}(\mathbb{S}(t) \psi)$ is the newly infective and recovered distribution. Hence, by the general results in [31] (see also in [31], Lemma 2.2),

$$
L(\psi):=\int_{0}^{\infty} V(S(t) \psi) \mathrm{d} t=V\left(\int_{0}^{\infty} S(t) \psi \mathrm{d} t\right),
$$

which is the total infective and recovered distribution, which is called the next infection operator. The spectral radius of $L$ is defined as $\mathfrak{R}_{0}$ of (14), i.e.,

$$
\mathfrak{R}_{0}:=r(L) .
$$

The following observation comes from [31].

Lemma 4. $\Re_{0}-1$ has the same sign as $\lambda(E)$ (or $\bar{\lambda}(U, \tau)$ ).

Generally, the above definition of $\Re_{0}$ is inconvenient for an application. For a special case, we compute it when all parameters are all independent of space variable, that is,

$$
\begin{aligned}
\lambda(x) & \equiv \lambda, \mu_{S}(x) \equiv \mu_{S}, \beta(x) \equiv \beta, a(x) \equiv a, b(x) \equiv b, \\
\mu_{I}(x) & \equiv \mu_{I}, k(x) \equiv k, r(x) \equiv r, \mu_{R}(x) \equiv \mu_{R}, m(x) \equiv m .
\end{aligned}
$$

Then (14) reduces to

$$
\begin{cases}\frac{\partial u_{1}}{\partial t}=d_{1} \Delta u_{1}+\lambda-\mu_{S} u_{1}-\frac{\beta u_{1} u_{2}}{1+\mathrm{au}_{1}+\mathrm{bu}_{2}}, & t>0, x \in \Omega \\ \frac{\partial u_{2}}{\partial t}=d_{2} \Delta u_{2}+\frac{\beta u_{1} u_{2}}{1+\mathrm{au}_{1}+\mathrm{bu} \mathrm{u}_{2}}-\left(\mu_{I}+k\right) u_{2}+\mathrm{ru}_{3}, & t>0, x \in \Omega \\ \frac{\partial u_{3}}{\partial t}=d_{3} \Delta u_{3}-\left(\mu_{R}+r\right) u_{3}+\int_{\Omega} G(\tau, x, y) \frac{\mathrm{ku}_{2}(t-\tau, y)}{1+\mathrm{mu}_{2}(t-\tau, y)} \mathrm{d} y, & t>0, x \in \Omega \\ \frac{\partial u_{1}(t, x)}{\partial n}=\frac{\partial u_{2}(t, x)}{\partial n}=\frac{\partial u_{3}(t, x)}{\partial n}=0, & t>0, x \in \partial \Omega\end{cases}
$$

Clearly, system (40) has the disease-free equilibrium $\left(\lambda / \mu_{S}, 0,0\right)$. The next generation operator $[L]$ of $(40)$ is given by

$$
[L](\psi):=\int_{0}^{\infty} V(S(t) \psi) \mathrm{d} t=V\left(\int_{0}^{\infty} S(t) \psi \mathrm{d} t\right), \quad \psi \in \mathbb{Y} \times \mathbb{Y},
$$

where

$$
V=\left(\begin{array}{cc}
\frac{\beta U}{1+a U} & r \\
k \int_{\Omega} G(\tau, x, y) \mathrm{d} y & 0
\end{array}\right) \text {, }
$$

and

$$
S(t) \psi=\left(\begin{array}{l}
\int_{\Omega} G_{2}(t, \cdot, y) \mathrm{d} y \psi_{2} \\
\int_{\Omega} G_{3}(t, \cdot, y) \mathrm{d} y \psi_{3}
\end{array}\right), \quad \psi \in \mathbb{Y} \times \mathbb{Y} .
$$

Recall that $G_{i}(t, \cdot, y)(i=2,3)$ are Green functions associated with $d_{2} \Delta-\left(\mu_{I}+k\right)$ and $d_{3} \Delta-\left(\mu_{R}+r\right)$ obeying the no-flux boundary condition. Then $\int_{\Omega} G_{2}(t, \cdot, y) d y=e^{-\left(\mu_{I}+k\right) t}$ and $\int_{\Omega} G_{3}(t, \cdot, y) d y=e^{-\left(\mu_{R}+r\right) t}$. For any $s>0$, we get

$$
\int_{\Omega} G(s, x, y) \mathrm{d} y=e^{-\left(\mu_{R}+r\right) s} .
$$

Then the next infection operator defined by (41) becomes 


$$
\begin{aligned}
{[L]\left(\begin{array}{l}
\psi_{2} \\
\psi_{3}
\end{array}\right) } & =\left(\begin{array}{cc}
\frac{\beta U}{1+\mathrm{aU}} & r \\
\mathrm{ke}^{-\left(\mu_{R}+r\right) \tau} & 0
\end{array}\right)\left(\begin{array}{cc}
\frac{1}{\mu_{I}+k} & 0 \\
0 & \frac{1}{\mu_{R}+r}
\end{array}\right)\left(\begin{array}{l}
\psi_{2} \\
\psi_{3}
\end{array}\right) \\
& =\left(\begin{array}{cc}
\frac{\beta U}{\left(\mu_{I}+k\right)(1+\mathrm{aU})} & \frac{r}{\mu_{R}+r} \\
\frac{\mathrm{ke}^{-\left(\mu_{R}+r\right) \tau}}{\mu_{I}+k} & 0
\end{array}\right)\left(\begin{array}{l}
\psi_{2} \\
\psi_{3}
\end{array}\right) .
\end{aligned}
$$

It follows that

$$
\left[\Re_{0}\right]=\frac{\beta U}{2\left(\mu_{I}+k\right)(1+a U)}+\sqrt{\frac{\beta^{2} U^{2}}{4\left(\mu_{I}+k\right)^{2}(1+\mathrm{aU})^{2}}+\frac{\mathrm{kre}^{-\left(\mu_{R}+r\right) \tau}}{\left(\mu_{I}+k\right)\left(\mu_{R}+r\right)}} .
$$

\section{Threshold Dynamics}

Theorem 2. For any $u_{0}=\phi \in C^{+}$, denote by $u(t, x, \phi)$ the solution of (14). The DFSS is globally attractive provided that $\mathfrak{R}_{0}<1$.

Proof. From Lemma 4, we know that $\lambda(E)<0$. Due to

$$
\lim _{\varepsilon \longrightarrow 0} \lambda(E+\varepsilon)=\lambda(E)<0,
$$

we can choose $\varepsilon_{0}>0$ where $\lambda\left(E+\varepsilon_{0}\right)<0$. Fixing $\varepsilon_{0}>0$, by Lemma 1 , and choosing $t_{0}=t_{0}(\phi)$ as $u_{1} \leq E(x)+\varepsilon_{0}, \forall t \geq t_{0}$, $x \in \bar{\Omega}$. It follows that

$$
\begin{cases}\frac{\partial u_{2}}{\partial t} \leq d_{2} \Delta u_{2}+\left(\frac{\beta(x)\left(E(x)+\varepsilon_{0}\right)}{1+a(x)\left(E(x)+\varepsilon_{0}\right)}-\mu_{I}(x)-k(x)\right) u_{2}+r(x) u_{3}, & t \geq t_{0}, x \in \Omega, \\ \frac{\partial u_{3}}{\partial t} \leq d_{3} \Delta u_{3}-\left(\mu_{R}(x)+r(x)\right) u_{3}+\int_{\Omega} G(\tau, x, y) k(y) u_{2}(t-\tau, y) \mathrm{d} y & t \geq t_{0}, x \in \Omega .\end{cases}
$$

From Lemma 3 , let $\psi_{0}$ be the strongly positive eigenfunction to $\bar{\lambda}\left(E+\varepsilon_{0}, \tau\right)<0$. Hence the linear system

$$
\begin{cases}\frac{\partial v_{1}}{\partial t}=d_{2} \Delta v_{1}+\left(\frac{\beta(x)\left(E(x)+\varepsilon_{0}\right)}{1+a(x)\left(E(x)+\varepsilon_{0}\right)}-\mu_{I}(x)-k(x)\right) v_{1}+r(x) v_{2}, & t>0, x \in \Omega \\ \frac{\partial v_{2}}{\partial t}=d_{3} \Delta v_{2}-\left(\mu_{R}(x)+r(x)\right) v_{2}+\int_{\Omega} G(\tau, x, y) k(y) v_{1}(t-\tau, y) \mathrm{d} y, & t>0, x \in \Omega \\ \frac{\partial v_{1}(t, x)}{\partial n}=\frac{\partial v_{2}(t, x)}{\partial n}=0, & x \in \partial \Omega\end{cases}
$$

has a solution $v(t, \cdot)=e^{\bar{\lambda}\left(E+\varepsilon_{0}, \tau\right) t} \psi_{0}(\cdot)$. Choose $\alpha>0$ large enough that

$$
\left(u_{2}(t, \cdot, \phi), u_{3}(t, \cdot, \phi)\right)^{T} \leq \alpha v(t, \cdot), \quad \forall t \in\left[t_{0}-\tau, t_{0}\right] .
$$




$$
\left(u_{2}(t, \cdot, \phi), u_{3}(t, \cdot, \phi)\right)^{T} \leq \alpha e^{\bar{\lambda}\left(E+\varepsilon_{0}, \tau\right) t} \psi_{0}(\cdot), \quad \forall t \geq t_{0},
$$

which implies that

$\lim _{t \rightarrow \infty}\left(u_{2}(t, x, \phi), u_{3}(t, x, \phi)\right)=(0,0)$, uniformly for $x \in \bar{\Omega}$.

Consequently, $u_{1}$-equation is asymptotic to (19). From the standard arguments for asymptotically autonomous semiflows (we refer to [32] [Corollary 4.3]), $\lim _{t \longrightarrow \infty} u_{1}(t, x, \phi)=E(x)$. This proves Theorem 2 .

Theorem 3. For each $u_{0}=\phi \in C^{+}$, denote by $u(t, x, \phi)$ the solution of (14). If $\mathfrak{R}_{0}>1$ and $\phi_{i}(0, \cdot) \equiv 0$ for $i=2,3$, then there is sufficiently small $\eta>0$ that

$$
\liminf _{t \longrightarrow \infty} \Gamma(t, x) \geq \eta \text {, uniformly for } x \in \bar{\Omega},
$$

where $\Gamma=u_{1}, u_{2}, u_{3}$, respectively. Further, (14) possesses at least one PSS $u^{*}(x)=\left(u_{1}^{*}(x), u_{2}^{*}(x), u_{3}^{*}(x)\right)$.

Proof. From Lemma 3, we get $\bar{\lambda}(U, \tau)>0$. Define

$$
\mathbb{W}_{0}=\left\{\phi \in C^{+}: \phi_{2}(0, \cdot) \equiv 0 \text { and } \phi_{3}(0, \cdot) \equiv 0\right\},
$$

and

$$
\partial \mathbb{W}_{0}=\frac{C^{+}}{\mathbb{W}_{0}}=\left\{\phi \in C^{+}: \phi_{2}(0, \cdot) \equiv 0 \text { or } \phi_{3}(0, \cdot) \equiv 0\right\} .
$$

In these settings, Lemma 2 tells us that $u_{2}(t, x, \phi)>0$ and $u_{3}(t, x, \phi)>0, \forall x \in \bar{\Omega}, t>0$; that is, $\Phi(t) \mathbb{W}_{0} \subset \mathbb{W}_{0}$. Define

$$
M_{\partial}:=\left\{\phi \in \partial \mathbb{W}_{0}: \Phi(t) \phi \in \partial \mathbb{W}_{0}, t \geq 0\right\} .
$$

In what follows, we prove two claims.

Claim 1. $\omega(\psi)=\{$ DFSS $\}, \forall \psi \in M_{\partial}$, where $\omega(\phi)$ is the omega limit set of the orbit $\Phi(t) \phi: \forall t \geq 0$.

In fact, for each $\psi \in M_{\partial}, \Phi(t) \psi \in \partial \mathbb{W}_{0}, \forall t \geq 0$. Obviously, for each $t \geq 0$, either $u_{2}(t, \cdot, \psi) \equiv 0$ or $u_{3}(t, \cdot, \psi) \equiv 0$. We next confirm the claim in two cases. If $u_{2}(t, \cdot, \psi) \equiv 0$ for all $t \geq 0$, from Lemma 1, we directly have $\lim _{t \rightarrow \infty} u_{1}(t, x, \psi)=E(x)$. As to $u_{3}$-equation, it gives that $\lim _{t \rightarrow \infty} u_{3}(t, x, \psi)=0$. If $u_{2}\left(t_{0}, \cdot, \psi\right) \equiv 0$ for some $t_{0} \geq 0$, then $u_{2}(t, x, \psi)>0, \forall t$ $>t_{0}, x \in \bar{\Omega}$ (from Lemma 2 ). Hence, $u_{3}(t, \cdot, \psi) \equiv 0, \forall t \geq t_{0}$. Then from $u_{2}$-equation of (14), we get $\lim _{t \rightarrow \infty} u_{2}(t, x, \psi)=0$. Consequently, with the help of the standard arguments of asymptotically autonomous semiflows, $u_{1}$ satisfies $\lim _{t \longrightarrow \infty} u_{1}(t, x, \psi)=E(x)$.

Claim 2. $\limsup _{t \rightarrow \infty}\|\Phi(t)(\phi)-\mathrm{DFSS}\| \geq \delta_{0}$, for all $\phi \in \mathbb{W}_{0}$.

We will prove this claim by way of contradiction. If limsup $_{t \rightarrow \infty}\left\|\Phi(t)\left(\phi_{0}\right)-\mathrm{DFSS}\right\|<\delta_{0}$ for some $\phi_{0} \in \mathbb{W}_{0}$, we can choose large enough $t_{*}>0$ as $u_{1}\left(t, \cdot, \phi_{0}\right)>E(x)-\delta_{0}$ and $u_{2}\left(t, \cdot, \phi_{0}\right)<\delta_{0}, \forall t>t_{*}, x \in \bar{\Omega}$. Hence, we have

$$
\left\{\begin{array}{l}
\frac{\partial u_{2}}{\partial t} \geq d_{2}(t, x) \Delta u_{2}+\left(\frac{\beta(x)\left(E(x)-\delta_{0}\right)}{1+a(x)\left(E(x)-\delta_{0}\right)+b(x) \delta_{0}}-\mu_{I}(x)-k(x)\right) u_{2}+r(x) u_{3} \\
\frac{\partial u_{3}}{\partial t} \geq d_{3} \Delta u_{3}-\left(\mu_{R}(x)+r(x)\right) u_{3}+\int_{\Omega} G_{2}(\tau, x, y) \frac{k(y) u_{2}(t-\tau, y)}{1+m(y) \delta_{0}} \mathrm{~d} y
\end{array}\right.
$$

for $\forall t>t_{*}, x \in \Omega$. Denote by $\varphi_{0}$ the positive eigenvector to $\bar{\lambda}\left(E-\delta_{0}, \tau\right)$. Then system

$$
\left\{\begin{array}{l}
\frac{\partial v_{1}}{\partial t}=d_{2} \Delta v_{1}+\left(\frac{\beta(x)\left(E(x)-\delta_{0}\right)}{1+a(x)\left(E(x)-\delta_{0}\right)+b(x) \delta_{0}}-\mu_{I}(x)-k(x)\right) v_{1}+r(x) v_{2}, \\
\frac{\partial v_{2}}{\partial t}=d_{3} \Delta v_{2}-\left(\mu_{R}(x)+r(x)\right) v_{2}+\int_{\Omega} G_{2}(\tau, x, y) \frac{k(y) v_{1}(t-\tau, y)}{1+m(y) \delta_{0}} \mathrm{~d} y, \\
\frac{\partial v_{1}(t, x)}{\partial n}=\frac{\partial v_{2}(t, x)}{\partial n}=0, \quad x \in \partial \Omega,
\end{array}\right.
$$




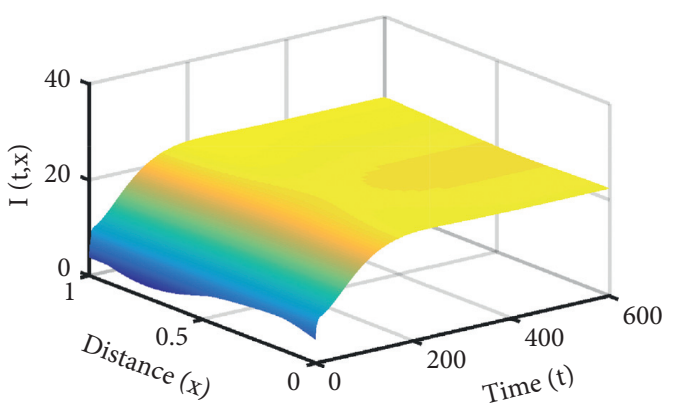

(a)

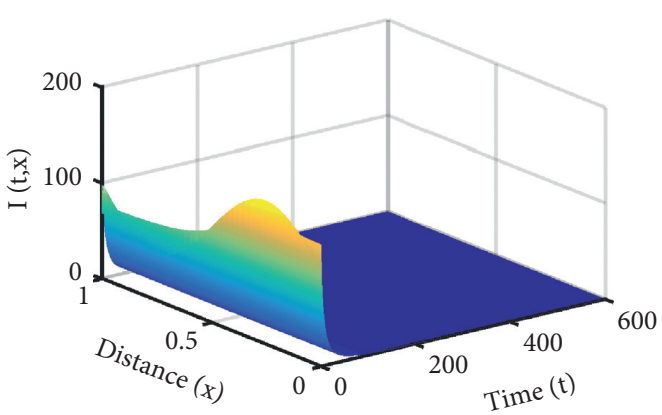

(b)

Figure 1: The time evolution of the densities of $I(t, x)$ for different $\mathfrak{R}_{0}$. (a) $\mathfrak{R}_{0}>1$; (b) $\mathfrak{R}_{0}<1$.

possesses the solution $v(t, \cdot)=e^{\bar{\lambda}\left(U-\delta_{0}, \tau\right) t} \varphi_{0}(\cdot)$. Since $u_{2}\left(t, x, \phi_{0}\right) \gg 0$ and $u_{3}\left(t, x, \phi_{0}\right) \gg 0$, we can choose small enough $\xi>0$ that

$$
\left(u_{2}\left(t, \cdot, \phi_{0}\right), u_{3}\left(t, \cdot, \phi_{0}\right)\right)^{T} \geq \xi v(t, x), \quad \forall t \in\left[t_{1}-\tau, t_{1}\right], \quad x \in \bar{\Omega} .
$$

By (57), together with the comparison principle, we directly obtain

$$
\left(u_{2}\left(t, \cdot, \phi_{0}\right), u_{3}\left(t, \cdot, \phi_{0}\right)\right)^{T} \geq \xi e^{\bar{\lambda}\left(E-\delta_{0}, \tau\right) t} \varphi_{0}(\cdot), \quad \forall t>t_{1}, \quad x \in \bar{\Omega} .
$$

By choosing a small enough $\delta_{0}$ that $\bar{\lambda}\left(U-\delta_{0}, \tau\right)>0$, it implies that $u_{2}\left(t, \cdot, \phi_{0}\right)$ and $u_{3}\left(t, \cdot, \phi_{0}\right)$ are unbounded, a contradiction. This proves Claim 2 .

Let $D(\cdot): C^{+} \longrightarrow \mathbb{R}_{+}$be a generalized distance function defined by

$$
D(\phi)=\min \left\{\min _{x \in \bar{\Omega}} \phi_{2}(0, x), \min _{x \in \bar{\Omega}} \phi_{3}(0, x)\right\}, \quad \forall \phi \in C^{+} .
$$

Clearly, $D^{-1}(0, \infty) \subset \mathbb{W}_{0}$. By Lemma $2, D(\cdot)$ enjoy the property that if $D(\phi)=0$ and $\phi \in \mathbb{W}_{0}$ or $D(\phi)>0$, then $D(\Phi(t) \phi)>0$ for all $t>0$ (see [33]). Notice that any forward semiflow of $\Phi(t)$ in $M_{\partial}$ converges to DFSS, and DFSS is isolated in $C^{+}$with $W^{s}$ (DFSS) $\cap \mathbb{W}_{0}=0$. Here $W^{s}$ (DFSS) represents the stable set of DFSS. Moreover, no cycle is formed in $M_{\partial}$ from DFSS to DFSS. In summary, by [19] [Theorem 2.3], we arrive at the conclusion that, for any $\phi \in \mathbb{W}_{0}$, choosing $\eta>0$ small enough can give $\min \{D(\psi): \psi \in \omega(\phi)\}>\eta$. Hence,

$$
\liminf _{t \longrightarrow \infty} u_{2}(t, \cdot) \geq \eta, \liminf _{t \longrightarrow \infty} u_{3}(t, \cdot) \geq \eta, \quad \forall x \in \bar{\Omega} .
$$

On the other hand, from Lemma 2 and letting $\eta$ small enough, we obtain that $\liminf _{t \rightarrow \infty} u_{1}(t, x) \geq \eta, \forall x \in \bar{\Omega}$. This proves the first part of Theorem 3.

By [34] [Theorem 3.7 and Remark 3.10], $\Phi(t): \mathbb{W}_{0} \longrightarrow \mathbb{W}_{0}$ has a global compact attractor. Further from [34] [Theorem 4.7], the semiflow $\Phi(t)$ admits a PSS $u^{*}(x) \in \mathbb{W}_{0}$. Lemma 2 ensures that $u^{*}(x)$ is a strictly PSS of (14).

\section{Simulations}

In this section, we mainly focus on the effects of the spatial heterogeneity and the diffusion rates of individuals on the disease dynamics. For simplicity, we assume that $\Omega=(0,1)$. Let $d_{S}=0.3, \quad d_{I}=0.1, \quad d_{R}=0.2, \quad \tau=0.4, \quad \lambda(x)=5$, $\mu_{S}(x)=0.01, \quad \mu_{I}(x)=0.05, \quad \mu_{R}(x)=0.15, \quad r(x)=0.2$, $a(x)=1, b(x)=1, \quad m(x)=0.1, \quad \beta(x)=0.15 \times(1+$ $0.5 \cos (2 \pi x)), k(x)=0.1 \times(1+0.2 \sin (2 \pi x))$. Then $\mathfrak{R}_{0}=$ 1.2644 and the disease is persistent (see Figure 1(a) with initial values $S(t, x)=20 \times(1+0.2 \sin (2 \pi x)), \quad I(t, x)=$ $5 \times(1+0.5 \sin (2 \pi x)), \quad R_{r}(t, x)=10 \times(1+0.2 \cos (2 \pi x))$ for $(t, x) \in[-\tau, 0] \times(0,1))$. Assume that $\beta(x)=0.09 \times(1$ $+0.5 \cos (2 \pi x)), \quad I(t, x)=100 \times(1+0.5 \sin (2 \pi x))$ for $(t, x) \in[-\tau, 0] \times(0,1))$, and other parameters and initial values are the same as those in Figure 1(a); then $\boldsymbol{R}_{0}=0.9496$ and the disease is extinct (see Figure 1(b)).

Now, we show the influence of diffusion rate on $\mathfrak{R}_{0}$ and the number of infected individuals. Let $\beta(x)=0.45 \times(1+0.5 \cos (2 \pi x)), \quad k(x)=0.3 \times(1+0.2$ $\sin (2 \pi x))$, and let other parameters be the same as those in Figure 1 except for $d_{I}$; it is shown that $\mathfrak{R}_{0}$ decreases as $d_{I}$ increases (see Figure 2(a)). Furthermore, let $\beta(x)=0.27 \times$ $(1+0.5 \cos (2 \pi x))$ and other parameters be the same as those in Figure 1 except for $d_{I}$; we find that the number of infected individuals $I(t, x)$ at steady state also decreases as $d_{I}$ increases (see Figure 2(b) with the same initial values as those in Figure 1).

Next, we show the influence of spatial heterogeneity on $\mathfrak{R}_{0}$. Let $\beta(x)=0.45 \times(1+0.5 \sin (2 \pi x))$, and let other parameters be the same as those in Figure 1 except for $k(x)$; then $\mathfrak{R}_{0}$ is nonincreasing as the heterogeneity parameter $c$ of $k(x)$ increases (see Figure 3(a) with $k(x)=0.3 \times(1+0.8 c \sin (2 \pi x)))$. However, if we take $\beta(x)=0.45 \times(1+0.5 \cos (2 \pi x))$ and let other parameters be the same as those in Figure $3(\mathrm{a})$, then $\mathfrak{R}_{0}$ is nondecreasing as the heterogeneity parameter $c$ of $k(x)$ increases (see Figure 3(b) with $k(x)=0.3 \times(1$ $+0.8 c \sin (2 \pi x)))$. Therefore, the effects of the spatial heterogeneity may be dependent on the actual environment. 

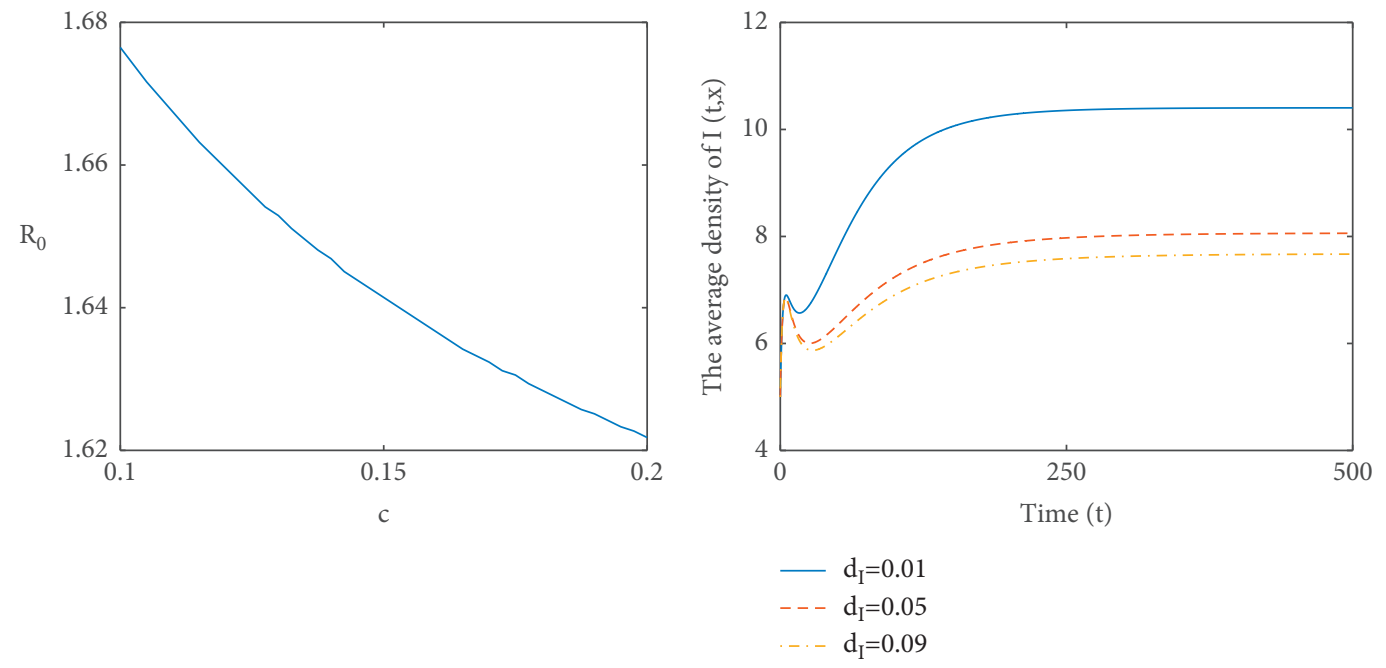

(a)

(b)

Figure 2: The influence of diffusion rate on infected individuals. (a) $d_{I}=0.1 c$. (b) The evolution of $I(t, x)$.

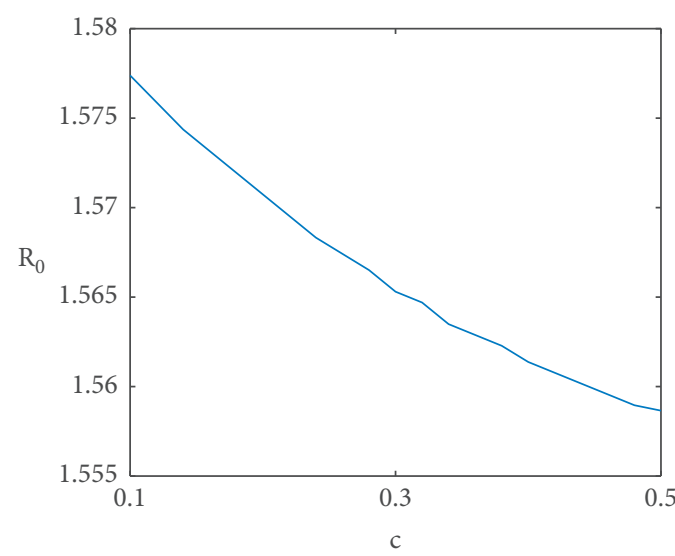

(a)

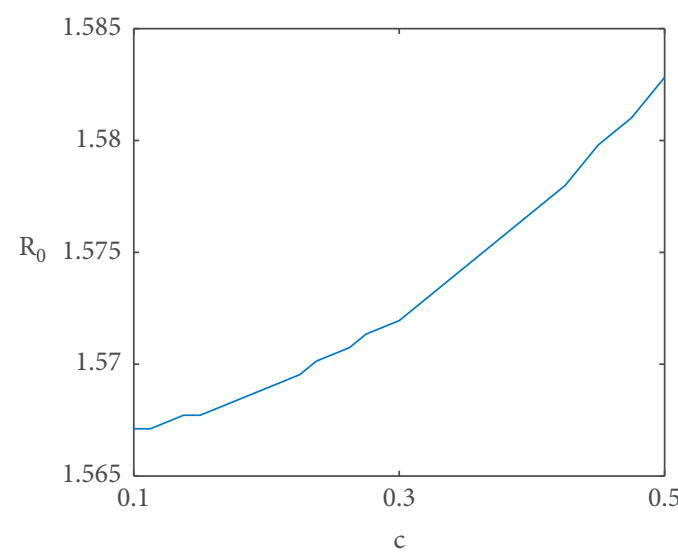

(b)

Figure 3: The influence of spatial heterogeneity on $\mathfrak{R}_{0}$ with $k(x)=0.3 \times(1+0.8 c \sin (2 \pi x))$. (a) $\beta(x)=0.45 \times(1+0.5 \sin (2 \pi x))$; (b) $\beta(x)=0.45 \times(1+0.5 \cos (2 \pi x))$.

\section{Conclusion and Discussion}

This paper performs a complete analysis on the thresholdtype dynamics of a diffusive herpes model, like in [10] where the spatial movement of mosquitoes in EIP will result in nonlocal infection. Here we introduce a relapse age to be a continuous variable and nonlinear recovery rate. We conducted a complete analysis of model (14) by adopting a fixed relapse period, which can be regarded as a continuous work of [10]. In Theorem 1, we confirmed that (14) admits a unique solution $u(t, \phi)$ on $[0, \infty)$. Consequently, the solution semiflow generated by the solution of (14) possesses a global attractor in $C^{+}$. By using the next generation operator approach, we characterize $\boldsymbol{R}_{0}$ as its spectral radius. To build up the relation between $\boldsymbol{R}_{0}$ and the principle eigenvalue of associated eigenvalue problem, we establish the relation between the principle eigenvalue of (30) and the principle eigenvalue of (33). To proceed further, we consider the special case where parameters are all independent of $x$ and calculate $\mathfrak{R}_{0}$ explicitly. By utilizing the principal eigenvalue of the associated eigenvalue problem or equivalently by $\mathfrak{R}_{0}$, we establish the threshold-type dynamics of the model in the sense that the DFSS is globally attractive provided that $\mathfrak{R}_{0}<1$, while if $\mathfrak{R}_{0}>1$, system (14) is uniformly persistent and (14) possesses at least one PSS $u^{*}(x)=\left(u_{1}^{*}(x), u_{2}^{*}(x), u_{3}^{*}(x)\right)$. This threshold-type result implies that, under the changing of the sign of $\mathfrak{R}_{0}-1$, the herpes will be extinct or close to the epidemic value. From the explicit expression of $\left[\mathfrak{R}_{0}\right]$ in (46), we know that it is closely related to the parameters. Compared to $\mathfrak{R}_{0}$ defined by the spectral radius of next generation operator, it is a challenging to analyze the impact of parameters on $\mathfrak{R}_{0}$, if it is not impossible. On the other hand, $\boldsymbol{R}_{0}$ is related to the principal eigenvalue of an elliptic system with two infected components, which makes the analysis more difficult. We perform the numerical simulations to verify the theoretical 
results and study the effect of the spatial heterogeneity in one-dimensional space $\Omega=(0,1)$. Threshold-type results are illustrated in Figure 1. In Figure 2, we can see the influence of diffusion rate on $\mathfrak{R}_{0}$ and the number of infected individuals. Specifically, $\mathfrak{R}_{0}$ decreases as $d_{I}$ increases (see Figure 2(a)) and the number of infected individuals $I(t, x)$ at steady state also decreases as $d_{I}$ increases (see Figure 2(b)). Figure 3 demonstrates the influence of spatial heterogeneity on $\mathfrak{R}_{0}$. We found that, with different $\beta(x), \mathfrak{R}_{0}$ is nonincreasing or nondecreasing in terms of $k(x)$. Therefore, the effects of the spatial heterogeneity may be dependent on the actual environment.

\section{Data Availability}

This paper is a theoretical research and therefore there is no underlying data.

\section{Conflicts of Interest}

The authors declare that they have no conflicts of interest.

\section{Acknowledgments}

Y. Lu was supported by Natural Science Foundation of Heilongjiang Province (A2018009) and University Nursing Program for Young Scholars with Creative Talents in Heilongjiang Province (UNPYSCT-2018206). D. Ji was supported by the National Natural Science Foundation of China (11711107).

\section{References}

[1] T. Hart, Microterrors, Firefly Books, Richmond Hill, Canada, 2004.

[2] S. Blower, "Modelling the genital herpes epidemic," Herpesviridae, vol. 11, no. Suppl. 3, p. 138A, 2004.

[3] S. M. Blower, T. C. Porco, and G. Darby, "Predicting and preventing the emergence of antiviral drug resistance in HSV-2," Nature Medicine, vol. 4, no. 6, pp. 673-678, 1998.

[4] H. N. Moreira and W. Yuquan, "Classroom note:global sm $S \longrightarrow I \longrightarrow R \longrightarrow I$ model," SIAM Review, vol. 39, no. 3, pp. 496-502, 1997.

[5] D. Tudor, "A deterministic model for herpes infections in human and animal populations," SIAM Review, vol. 32, no. 1, pp. 136-139, 1990.

[6] G. Smith and B. T. Grenfell, "Population biology of pseudorabies in swine," American Journal of Veterinary Research, vol. 51, pp. 148-155, 1990.

[7] P. van den Driessche and X. Zou, "Modeling relapse in infectious diseases," Mathematical Biosciences, vol. 207, no. 1, pp. 89-103, 2007.

[8] Y. Cai, X. Lian, Z. Peng, and W. Wang, "Spatiotemporal transmission dynamics for influenza disease in a heterogenous environment," Nonlinear Analysis: Real World Applications, vol. 46, pp. 178-194, 2019.

[9] Z. Guo, F.-B. Wang, and X. Zou, "Threshold dynamics of an infective disease model with a fixed latent period and nonlocal infections," Journal of Mathematical Biology, vol. 65, no. 6-7, pp. 1387-1410, 2012.
[10] Y. Lou and X.-Q. Zhao, “A reaction-diffusion malaria model with incubation period in the vector population," Journal of Mathematical Biology, vol. 62, no. 4, pp. 543-568, 2011.

[11] J. Wang and J. Wang, "Analysis of a reaction-diffusion cholera model with distinct dispersal rates in the human population," Journal of Dynamics and Differential Equations, vol. 33, no. 1, pp. 549-575, 2021.

[12] J. Wang and $\mathrm{X}$. Wu, "Dynamics and profiles of a diffusive cholera model with bacterial $\mathrm{h}$ and distinct dispersal rates," Journal of Dynamics and Differential Equations, 2021.

[13] J. Wang and R. Cui, "Analysis of a diffusive host-pathogen modelwith standard incidence and distinct dispersal rates," Advances in Nonlinear Analysis, vol. 10, pp. 922-951, 2021.

[14] X. Guo, J. Chen, A. Azizi, J. Fewell, and Y. Kang, "Dynamics of social interactions, in the flow of information and disease spreading in social insects colonies: effects of environmental events and spatial heterogeneity," Journal of Theoretical Biology, vol. 492, p. 110191, 2020.

[15] Z.-G. Guo, G.-Q. Sun, Z. Wang, Z. Jin, L. Li, and C. Li, "Spatial dynamics of an epidemic model with nonlocal infection," Applied Mathematics and Computation, vol. 377, p. 125158, 2020.

[16] G.-Q. Sun, M. Jusup, Z. Jin, Y. Wang, and Z. Wang, "Pattern transitions in spatial epidemics: mechanisms and emergent properties," Physics of Life Reviews, vol. 19, pp. 43-73, 2016.

[17] $\mathrm{Z}$. Xu and C. Ai, "Traveling waves in a diffusive influenza epidemic model with vaccination," Applied Mathematical Modelling, vol. 40, no. 15-16, pp. 7265-7280, 2016.

[18] F. P. Agouanet, I. Tankam-Chedjou, R. M. Etoua, and J. J. Tewa, "Mathematical modelling of Banana Black Sigatoka disease with delay and seasonality," Applied Mathematical Modelling, vol. 99, pp. 380-399, 2021.

[19] G.-Q. Sun, M.-T. Li, J. Zhang, W. Zhang, X. Pei, and Z. Jin, "Transmission dynamics of brucellosis: mathematical modelling and applications in China," Computational and Structural Biotechnology Journal, vol. 18, pp. 3843-3860, 2020.

[20] Q. Richard, M. Choisy, T. Lefèvre, and R. Djidjou-Demasse, "Human-vector malaria transmission model structured by age, time since infection and waning immunity," Nonlinear Anal.: RWA, vol. 63, Article ID 103393, 2021.

[21] P. Wu and H. Zhao, "Dynamical analysis of a nonlocal delayed and diffusive HIV latent infection model with spatial heterogeneity," Journal of the Franklin Institute, vol. 358, no. 10, pp. 5552-5587, 2021.

[22] K. Gaythorpe and B. Adams, "Disease and disaster: optimal deployment of epidemic control facilities in a spatially heterogeneous population with changing behaviour," Journal of Theoretical Biology, vol. 397, pp. 169-178, 2016.

[23] Beddington, "Mutual interference between parasites or predators and its effect on searching efficiency," Journal of Animal Ecology, vol. 44, no. 1, pp. 331-340, 1975.

[24] J. A. J. Metz and O. Diekmann, The Dynamics of Physiologically Structured Populations, J. A. J. Metz and O. Diekmann, Eds., Springer, New York, 1986.

[25] W. Wang and X.-Q. Zhao, "A nonlocal and time-delayed reaction-diffusion model of dengue transmission," SIAM Journal on Applied Mathematics, vol. 71, no. 1, pp. 147-168, 2011.

[26] H. L. Smith, "Monotone dynamical systems: an introduction tothetheory of competitiveand cooperative systems," Amer. Math. Soc. Math. Surveys and Monographs, vol. 41, 1995.

[27] R. H. Martin and H. L. Smith, "Abstract functional-differential equations and reaction-diffusion systems," Transactions 
of the American Mathematical Society, vol. 321, no. 1, pp. 1-44, 1990.

[28] J. Wu, Theory and Applications of Partial Functional Differential Equations, Springer, New York, US, 1996.

[29] J. Hale, Asymptotic Behavior of Dissipative Systems, American Mathematical Society, Providence, 1988.

[30] H. R. Thieme and X.-Q. Zhao, "A non-local delayed and diffusive predator-prey model," Nonlinear Analysis: Real World Applications, vol. 2, no. 2, pp. 145-160, 2001.

[31] Thieme, "Spectral bound and reproduction number for infinite-dimensional population structure and time heterogeneity," SIAM Journal on Applied Mathematics, vol. 70, no. 1, pp. 188-211, 2009.

[32] H. R. Thieme, "Convergence results and a Poincare-Bendixson trichotomy for asymptotically autonomous differential equations," Journal of Mathematical Biology, vol. 30, pp. 755-763, 1992.

[33] H. L. Smith and X.-Q. Zhao, "Robust persistence for semidynamical systems," Nonlinear Analysis: Theory, Methods \& Applications, vol. 47, no. 9, pp. 6169-6179, 2001.

[34] P. Magal and X.-Q. Zhao, "Global attractors and steady states for uniformly persistent dynamical systems," SIAM Journal on Mathematical Analysis, vol. 37, no. 1, pp. 251-275, 2005. 